\title{
siRNA targeting of Trop2 suppresses the proliferation and invasion of lung adenocarcinoma $\mathrm{H} 460$ cells
}

\author{
XIAO-YAN GAO ${ }^{1}$, YE-HAN ZHU ${ }^{2}$, LI-XIN ZHANG ${ }^{3}$, HUI-YU LU ${ }^{1}$ and AI-GUI JIANG ${ }^{1}$ \\ ${ }^{1}$ Department of Respiratory Medicine, Taizhou People's Hospital Affiliated to Medical College of Nantong University, \\ Taizhou, Jiangsu 225300; ${ }^{2}$ Department of Respiratory Medicine, The First Affiliated Hospital, Soochow University, \\ Suzhou, Jiangsu 215006; ${ }^{3}$ Institute of Clinical Medicine, Taizhou People's Hospital Affiliated \\ to Medical College of Nantong University, Taizhou, Jiangsu 225300, P.R. China
}

Received September 2, 2014; Accepted May 1, 2015

DOI: $10.3892 / \mathrm{etm} .2015 .2530$

\begin{abstract}
The aim of the present study was to investigate the effect of the small interfering RNA (siRNA)-induced inhibition of the Trop2 gene on the proliferation and invasion of lung adenocarcinoma H460 cells. A recombinant adenovirus expression vector, which contained siRNA targeting open reading frames for Trop2 (rAd5-siTrop2), was transfected into lung adenocarcinoma $\mathrm{H} 460$ cells. Three groups were included in the study, namely the Ctrl (non-transfected control), rAd5-siCtrl (native control) and rAd5-siTrop2 (knockdown Trop2 gene) groups. The mRNA and protein expression levels of Trop2 were detected using quantitative polymerase chain reaction and western blot analysis, respectively. In addition, the expression levels of cyclin Dl and phospho-extracellular signal regulated kinase (p-ERK)-1 were detected using western blot analysis. The effects of Trop2 inhibition on the proliferation and invasion of lung adenocarcinoma $\mathrm{H} 460$ cells were investigated using a 3-(4,5-dimethylthiazol-2-yl)-2,5-diphenyltetrazolium bromide and Transwell assay. Trop2-targeted siRNA recombinant plasmids were successfully constructed. The recombinant adenovirus vector, rAd5-siTrop2, significantly downregulated the mRNA and protein expression levels of Trop2 in the lung adenocarcinoma H460 cells, with cyclin D1 and p-ERK-1 expression downregulated simultaneously. In addition, following the silencing of Trop2, the proliferation and invasion rates of the lung adenocarcinoma H460 cells were reduced. Therefore, the results indicated that Trop2 serves a key func-
\end{abstract}

Correspondence to: Dr Ai-Gui Jiang, Department of Respiratory Medicine, Taizhou People's Hospital Affiliated to Medical College of Nantong University, 210 Yingchun Road, Taizhou, Jiangsu 225300, P.R. China

E-mail:jiangaigui@126.com

Professor Ye-Han Zhu, Department of Respiratory Medicine, The First Affiliated Hospital, Soochow University, 188 Shizi Road, Suzhou, Jiangsu 215006, P.R. China

Email: zhuyehansz@sina.com

Key words: Trop2, lung adenocarcinoma, small interfering RNA tion in the proliferation and invasion of lung adenocarcinoma H460 cells in vitro.

\section{Introduction}

Despite considerable progress in surgery, chemotherapy, radiotherapy and biological-targeted therapy, lung cancer remains a leading cause of cancer-associated mortality worldwide (1). A previous study indicated that the existing therapies function primarily to improve patient quality of life, and the overall five-year survival rate for lung tumors is $<15 \%$ (2). In addition, lung cancer is now the priority target for cancer prevention, early detection and therapy in China due to the increasing rate of incidence (3). As a consequence, research into novel prognostic biomarkers and therapeutic targets for lung cancer is increasing.

In particular, cancer gene therapy has become a focus of attention as an effective and specific treatment for lung cancer. Recent developments in molecular biological techniques and the improved understanding of the pathogenesis of lung cancer has revealed that certain cancer-associated genes may be used as effective targets for lung cancer gene therapy. Previous studies have identified that the genes for epidermal growth factor receptor (4), GA733 (5), echinoderm microtubule-associated protein-like 4-anaplastic lymphoma kinase (6), colloid osmotic pressure of serum, and lung fibroblast growth factor receptor 1 (closely related to FGFR1) (7) are associated with tumor growth and development, apoptosis, metastasis, cell proliferation and aggressiveness. These genes are potential intervention targets for cancer gene therapy, and a number of the genes have exhibited beneficial therapeutic effects following clinical application.

Trop2, also known as GA733-1, M1S1 or EGP-1, is a human trophoblast cell-surface antigen encoded by the TACSTD2 gene, which is located on human chromosome 1p32 (8). TACSTD2 encodes a 35-kDa type 1 transmembrane glycoprotein that contains a single transmembrane domain and 323 amino acids. Trop 2 is a calcium channel protein that is associated with the regulation of the intracellular calcium concentration (9). Furthermore, Trop2 serves a key function in the regulation of tumor proliferation by increasing the stability of cyclin D1 or by activating the extracellular signal-regulated 
kinase (ERK)-l-mitogen-activated protein kinase (MAPK) signaling pathway $(10,11)$. Trop2 was originally observed to be expressed at high levels on the surface of trophoblastic cells (12). However, previous studies have identified elevated expression of Trop2 in a number of human epithelial tumor types, including colorectal cancer (13), oral squamous cell carcinoma (14) pancreatic cancer (15) and lung cancer $(5,16,17)$, and elevated Trop2 expression is often associated with a poor prognosis.

Overactivation of the Trop2 gene has also been observed in a number of non-small cell lung cancer cell lines, including H1975, SPCA-1 and PC-9 (18). However, to the best of our knowledge, no prior studies in the English language literature have investigated the correlation between Trop2 expression and the proliferation and invasion of lung adenocarcinoma $\mathrm{H} 460$ cells in vitro. In the present study, the Trop2 gene was suppressed via RNA interference, and the expression levels of cyclin Dl and phospho (p)-ERK-1 were detected using western blot analysis. In addition, the effects of Trop2 silencing on the proliferation and invasion of lung adenocarcinoma $\mathrm{H} 460$ cells were investigated using a 3-(4,5-dimethylthiazol-2-yl)-2,5-diphenyltetrazolium bromide (MTT) and Transwell assay.

\section{Materials and methods}

Cell lines and cell culture. An H460 human lung cancer cell line was obtained from the American Type Culture Collection (Manassas, VA, USA). The cells were maintained in Dulbecco's modified Eagle's medium (DMEM; Beyotime Institute of Biotechnology, Beijing, China), supplemented with $10 \%$ fetal bovine serum (FBS; Gibco Life Technologies, Carlsbad, CA, USA), $100 \mathrm{U} / \mathrm{ml}$ penicillin and $100 \mu \mathrm{g} / \mathrm{ml}$ streptomycin (Beyotime Institute of Biotechnology), in a humidified incubator with $5 \% \mathrm{CO}_{2}$ at $37^{\circ} \mathrm{C}$.

Generation of the rAd5-siTrop2 RNA interference lentiviruses. Following the evaluation of the knockdown efficiencies of a number of small interfering RNA (siRNA) constructs, the following siRNA oligonucleotide (rAd5-siTrop2-siRNA) was selected to target the 21-bp interference sequence of the Trop2 gene: 5'-CTCCAAGTGTCTGCTGCTCAA-3'. The rAd5-siTrop2-siRNA sequences were as follows: Sense, 5'-ACACTTGGAGGTTTTGGCCACTGACTGACT CCAAGCTGCTGCTCAA-3', and antisense, 3'-CCTGTT GAGCAGCAGACTTGGAGGTCAGTCAGTGGCCAAAAC CTCCAAGTGTCTGCTGCTCAAC-5'. In addition, a non-specific siRNA (rAd5-siCtrl-siRNA) was synthesized as a native control. Sequences were chemically synthesized by Shanghai GenePharma Co., Ltd. (Shanghai, China). $\mathrm{H} 460$ cells were seeded into six-well plates at a density of $2 \times 10^{5}$ cells/well, and transfection was performed using electroporation (Gene Pulser MXcell ${ }^{\mathrm{TM}}$ Electroporation system; Bio-Rad Laboratories, Inc., Munich, Germany). Three groups were generated for the ensuing experiments, which included the Ctrl group (non-transfected group), rAd5-siCtrl group (cells infected with non-specific siRNA) and rAd5-siTrop2 group (cells infected with rAd5-siTrop2-siRNA). The cells were harvested for the subsequent experiments at $72 \mathrm{~h}$ post-transduction.
Reverse transcription-quantitative polymerase chain reaction (RT-qPCR). The sequences of the primers used for PCR were as follows: Trop2 forward, 5'-CCTCATCGCCGTCAT CGT-3, and reverse, 5'-CGGTTCCTTTCTCAACTCCC-3'; $\beta$-actin forward, 5'-CCTGGCACCCAGCACAAT-3', and reverse, 5'-GCCGATCCACACGGAGTACT-3'. Primers were synthesized by Shanghai Sangon Biotechnology Co., Ltd. (Shanghai, China). The expression levels of Trop2 were quantified using fluorescence RT-qPCR. Following the termination of each experiment, the cells were collected and the total RNA was isolated using TRIzol ${ }^{\circledR}$ reagent (Invitrogen Life Technologies, Carlsbad, CA, USA). cDNA was synthesized using a reverse transcription kit (Toyobo Co., Ltd., Osaka, Japan), according to the manufacturer's instructions. cDNA $(2.5 \mu \mathrm{l})$ was subjected to qPCR using SYBR-Green (Wuhan Boster Biological Engineering Co., Ltd., Wuhan, China) as the fluorescent reporter and 2.5X Real Master Mix (Wuhan Boster Biological Engineering Co., Ltd.). The specific gene primers for Trop2 and $\beta$-actin were amplified in separate reaction tubes, as described previously (19). Threshold cycle numbers of triplicate reactions were determined using ABI 7500 software (Applied Biosystems Life Technologies, Foster City, CA, USA) and the average was calculated. For each reaction, $\beta$-actin was included as an internal standard and the relative quantitative gene expression level was calculated using the $2^{-\Delta \Delta C \mathrm{Ct}}$ method.

Western blot analysis. Cells $\left(1 \times 10^{6}\right)$ were lysed on ice with 100-200 $\mu 1$ lysis buffer (Beyotime Institute of Biotechnology) for $5 \mathrm{~min}$. Following lysis the samples were centrifuged at $400 \mathrm{x}$ g for $5 \mathrm{~min}$, and cell extracts from the Ctrl, rAd5-siCtrl and rAd5-siTrop2 groups were collected. Subsequently, $50 \mu \mathrm{g}$ protein samples were electrophoresed on $12 \%$ SDS-polyacrylamide gels and transferred onto polyvinylidene fluoride membranes (Santa Cruz Biotechnology, Inc., Santa Cruz, CA, USA). The membranes were blocked in 5\% non-fat dry milk in Tris-buffered saline (TBS) for $1 \mathrm{~h}$ at room temperature, followed by incubation with various primary antibodies, including mouse monoclonal anti-human Trop2 (sc-80406; 1:1,000), mouse monoclonal anti-human cyclin D1 (sc-56302; 1:1,000) and rabbit anti-human-p-ERK-1 antibodies (sc-16982; 1:1,000; Santa Cruz Biotechnology, Inc.), overnight at $4^{\circ} \mathrm{C}$. Following three washes with TBS-Tween-20 (TBST), the membranes were incubated with $50 \mu 1$ horseradish peroxidase-conjugated $\mathrm{IgG}$ secondary antibody for $2 \mathrm{~h}$ at room temperature. The secondary antibodies used included peroxidase-labeled goat anti-rabbit IgG (BA1055) and rabbit anti-mouse IgG (BM2002; 1:2,000; Wuhan Boster Biological Engineering Co., Ltd.). In addition, each membrane was incubated with a mouse monoclonal anti-GAPDH antibody (sc-47724; Santa Cruz Biotechnology, Inc.) as a loading control. The membranes were washed three times with TBST, and bound antibodies were detected using enhanced chemiluminescence analysis (Beyotime Institute of Biotechnology, Haimen, China). The protein expression levels were quantitated via densitometry using Quantity One software (Bio-Rad Laboratories, Inc.).

Proliferation assay. Cell proliferation was evaluated using an MTT assay on days 1, 3 and 6. Cells from the three groups 

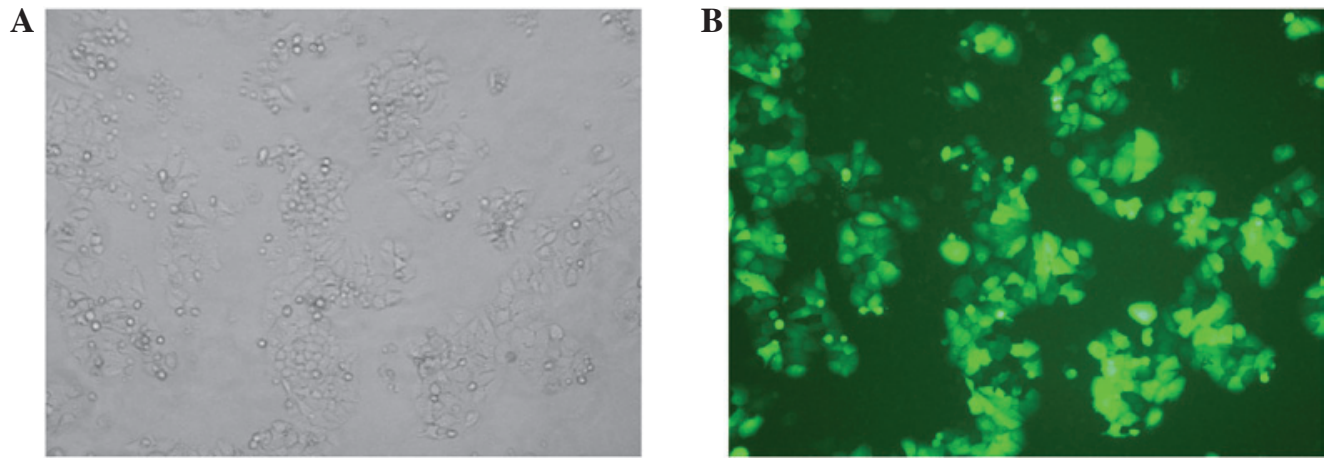

Figure 1. (A) Representative white field image of $\mathrm{H} 460$ cells and (B) corresponding fluorescence image showing the expression of green fluorescent protein in the same cell population, indicating the efficiency of the lentivirus infection (magnification, x100).

were plated onto 96-well plates (2,000 cells/well) and incubated with $20 \mu \mathrm{l}$ MTT (5 mg/ml; Sigma-Aldrich, St. Louis, MO, USA) for $4 \mathrm{~h}$ prior to collection. Subsequently, the culture medium was removed and $150 \mu \mathrm{l}$ dimethyl sulfoxide was added to the wells. Following vigorous shaking for $10 \mathrm{~min}$, the absorbance at $490 \mathrm{~nm}$ was measured using an enzyme immunoassay instrument (ETI-MAX3000; DiaSorin S.p.A, Saluggia, Italy). Six wells were analyzed for each group.

Transwell assay. Cell invasion was determined using a Transwell assay (Wuhan Boster Biological Engineering Co., Ltd.). Oligonucleotides were transfected into the cells according to the manufacturer's instructions. Following incubation for $48 \mathrm{~h}, 3 \times 10^{4}$ cells were transferred onto the top of the Matrigel-coated invasion chambers (BD Biosciences, San Jose, CA, USA) in serum-free DMEM, while DMEM containing $10 \%$ FBS was added to the lower chamber. After $24 \mathrm{~h}$, the non-invasive cells were removed, while the invasive cells were fixed with $95 \%$ ethanol, stained with $0.1 \%$ crystal violet (Nanjing Search Biotech, Co., Ltd., Nanjing, China) and photographed (magnification, x100; DM3000; Leica Microsystems, Mannheim, Germany). Each test consisted of three independent experiments.

Statistical analysis. Statistical analysis was performed using SPSS software, version 13.0 (SPSS, Inc., Chicago, IL, USA). All experiments were performed at least three times, and representative results are presented as the mean \pm standard deviation. Statistical analyses were performed using one-way analysis of variance, and comparisons among groups were conducted using the independent samples t-test, where $\mathrm{P}<0.05$ was considered to indicate a statistically significant difference.

\section{Results}

Efficiency of the infection assay for adenovirus transfection. For viral transduction, siRNA lentiviral vectors at a multiplicity of infection of 50 were added to dispersed H460 cells immediately following plating. After 3 days, green fluorescent protein (GFP) imaging confirmed the high efficiency of infection, which facilitated the follow-up experiments (Fig. 1). Subsequently, qPCR and western blot analysis assays were performed to detect the mRNA and protein expres-

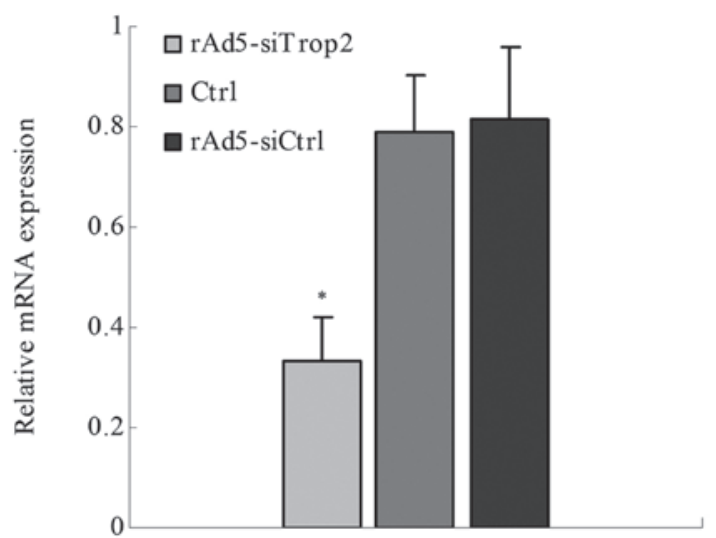

Figure 2. Reverse transcription-quantitative polymerase chain reaction assays indicated that the mRNA expression levels of Trop2 were significantly reduced in the rAd5-siTrop2-siRNA group when compared with the Ctrl and rAd5-Ctrl-siRNA groups. ${ }^{*} \mathrm{P}<0.01$, vs. Ctrl and rAd5-Ctrl-siRNA groups $(\mathrm{n}=3)$. siRNA, short interfering RNA; Ctrl, control.

sion levels of Trop2. The results indicated that the mRNA and protein expression levels of Trop2 were significantly decreased in the rAd5-siTrop2-siRNA group when compared with the Ctrl and rAd5-Ctrl-siRNA groups (Figs. 2 and 3). Thus, the results demonstrated the high knockdown efficiency of rAd5-siTrop2-siRNA in vitro.

Cyclin D1 and p-ERK-1 protein expression levels in the Ctrl, rAd5-Ctrl-siRNA and rAd5-siTrop2-siRNA groups. Western blot analyses were performed to detect the protein expression levels of cyclin D1 and p-ERK-1. In these assays, significant reductions were observed in the expression levels of cyclin D1 and p-ERK-1 in the rAd5-siTrop2-siRNA group when compared with the Ctrl and rAd5-Ctrl-siRNA groups (Fig. 3).

Trop2 knockdown mitigates the proliferation of $H 460$ cells. In the rAd5-siTrop2-siRNA group, the cells exhibited a reduced rate of proliferation when compared with the cells in the Ctrl or rAd5-Ctrl-siRNA group at day 3 after seeding $(\mathrm{P}<0.01$; Fig. 4).

Trop2 knockdown inhibits the migration of $H 460$ cells in vitro. Transwell migration assays were used to evaluate the effect of Trop2 knockdown on cell migration. The number of cells in 

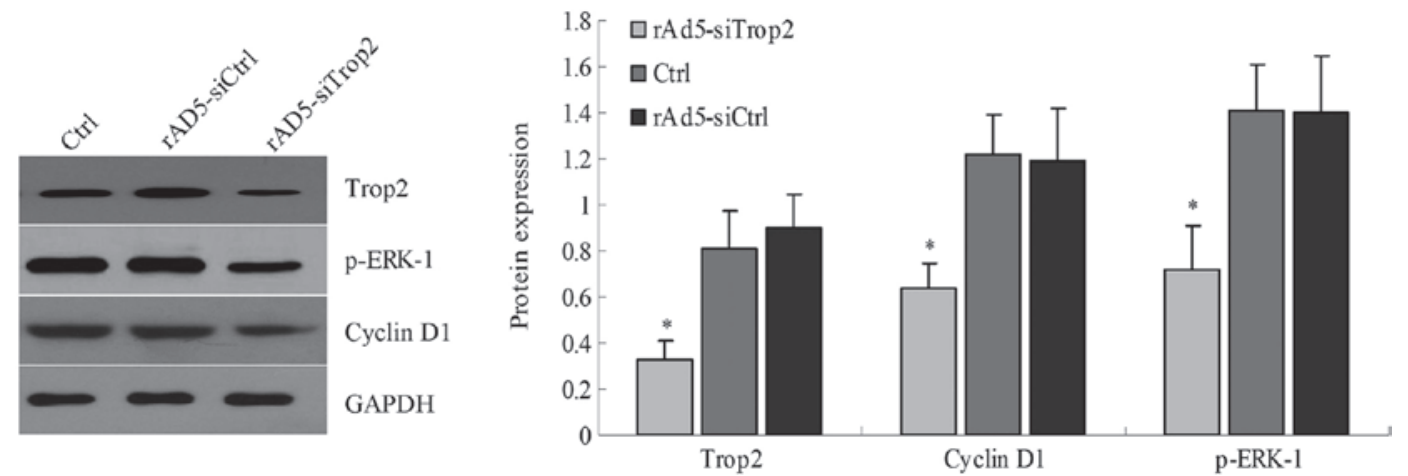

Figure 3. Western blot analysis assays revealed that the protein expression levels of Trop2, cyclin D1 and p-ERK-1 were significantly decreased in the rAd5-siTrop2-siRNA group when compared with the Ctrl and rAd5-Ctrl-siRNA groups. " $\mathrm{P}<0.01$, vs. Ctrl and rAd5-Ctrl-siRNA groups (n=3). Ctrl, control; siRNA, small interfering RNA; p-ERK, phospho-extracellular signal regulated kinase.

A
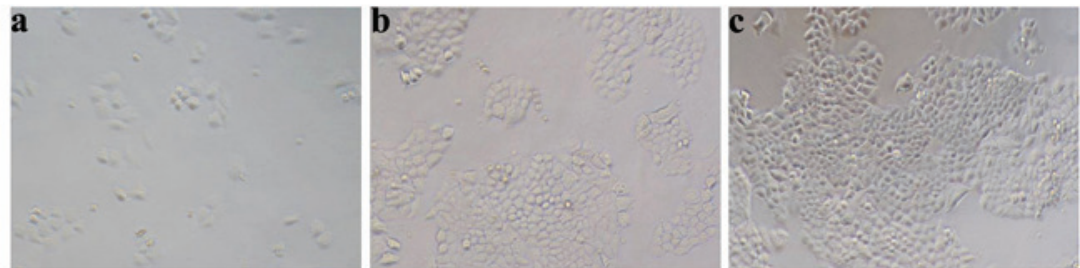

B
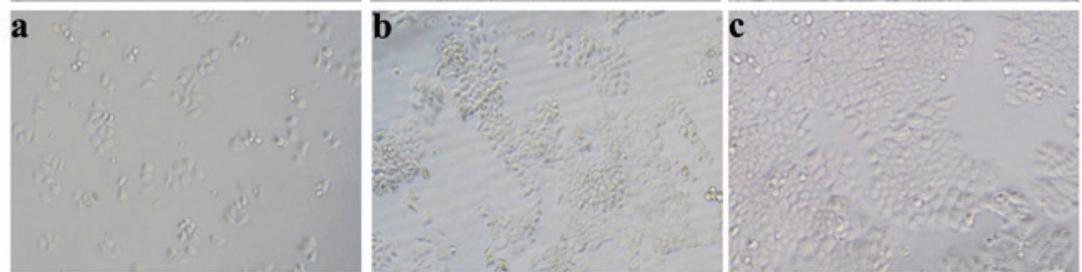

C
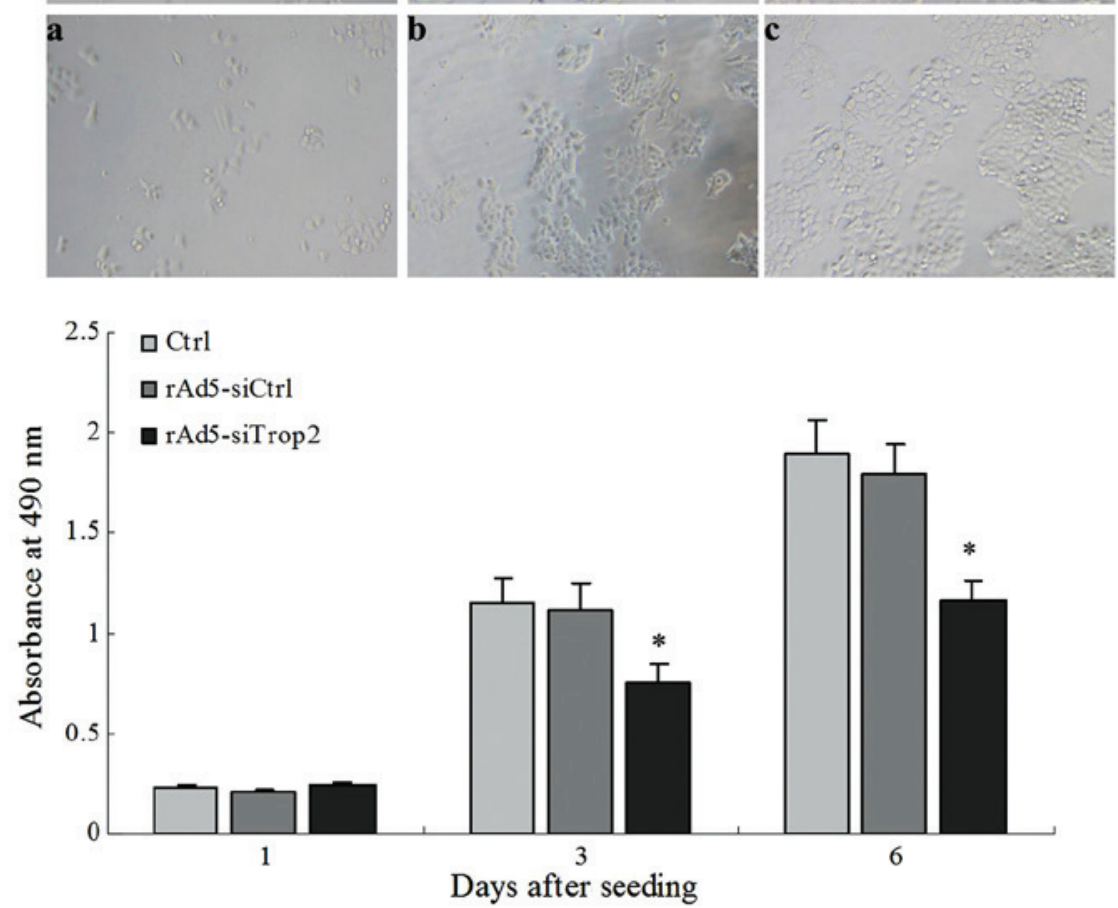

Figure 4. Cell proliferation was assessed using a 3-(4,5-dimethylthiazol-2-yl)-2,5-diphenyltetrazolium bromide assay in the (A) Ctrl, (B) rAd5-Ctrl-siRNA and (C) rAd5-siTrop2-siRNA groups. H460 cells exhibited a reduced proliferation rate at day 3 after seeding and silencing of the Trop2 gene. a, day 1 after seeding; b, day 3 after seeding; c, day 6 after seeding (magnification, x100). " $\mathrm{P}<0.01$, vs. Ctrl and rAd5-Ctrl-siRNA groups (n=3). siRNA, short interfering RNA; Ctrl, control.

the rAd5-siTrop2-siRNA group that traversed the micropore membrane was observed to be significantly reduced when compared with the rAd5-Ctrl-siRNA and Ctrl groups $(27 \pm 5$ vs. $56 \pm 7$ and $59 \pm 6$, respectively; $\mathrm{P}<0.01$; Fig. 5). These results indicated that the downregulation of Trop2 inhibits the migratory ability of $\mathrm{H} 460$ cells in vitro. 

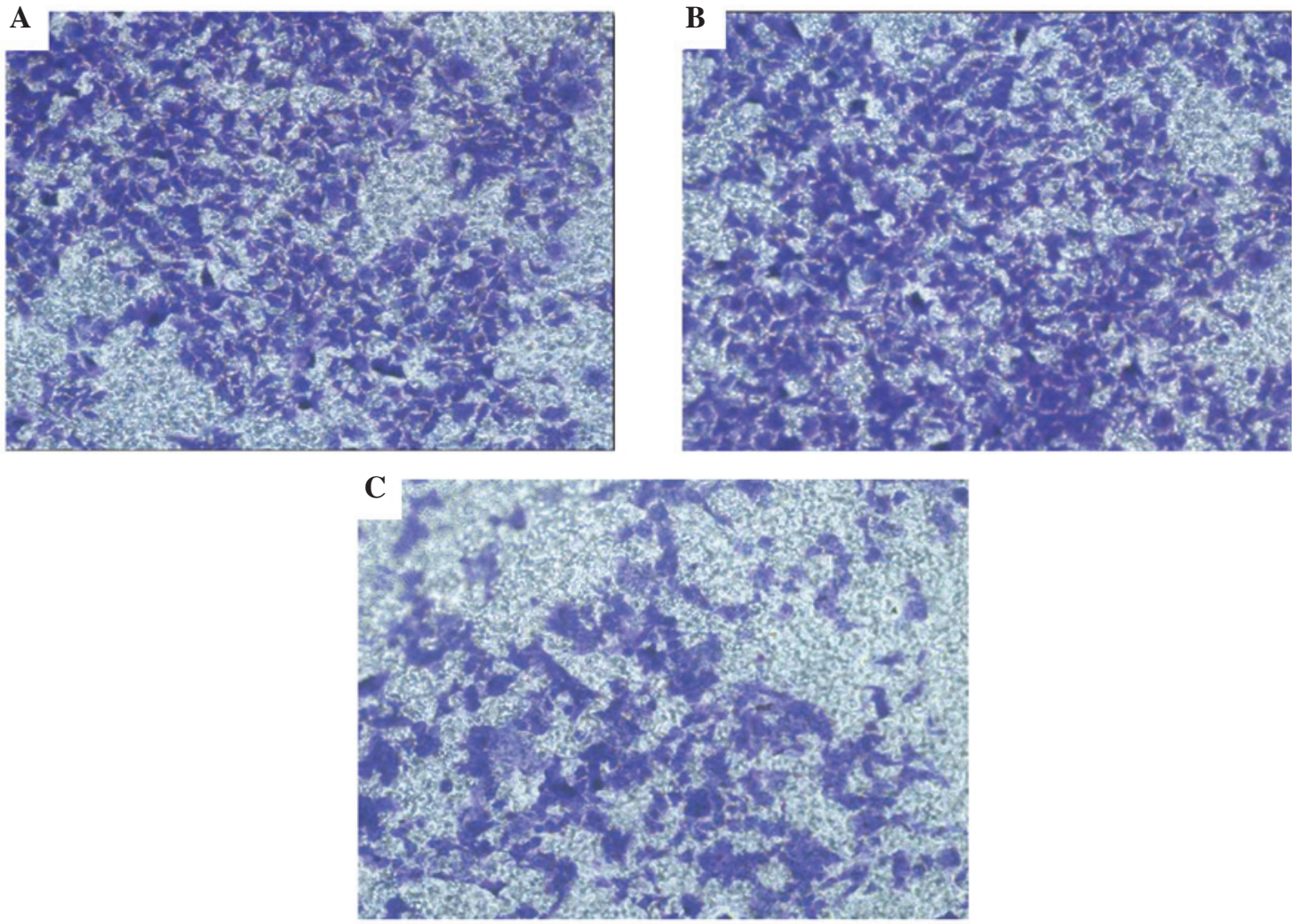

Figure 5. Transwell migration assays were used to assess the invasion ability of the cells in the (A) Ctrl, (B) rAd5-Ctrl-siRNA and (C) rAd5-siTrop2-siRNA groups. The results revealed that the number of cells in the rAd5-siTrop2-siRNA group that traversed the micropore membrane was reduced compared with the rAd5-Ctrl-siRNA and Ctrl groups (27 \pm 5 vs. 56 \pm and 59 \pm 6 , respectively; $\mathrm{P}<0.01 ; \mathrm{n}=3$ ) (magnification, $\mathrm{x} 100)$. siRNA, short interfering RNA; Ctrl, control

\section{Discussion}

Trop2 is a transmembrane glycoprotein that was initially observed to be expressed at high levels on the surface of trophoblastic cells (12). Trop2 is a human trophoblast cell-surface antigen encoded by the TACSTD2 gene on human chromosome $1 \mathrm{p} 32$ (8), and has been demonstrated to promote the proliferation of pancreatic and intestinal tumor cells (20). However, the function of Trop2 in the proliferation and differentiation of human lung adenocarcinoma H460 cells is yet to be fully elucidated. In the present study, siRNA technology was used to downregulate the expression of Trop2 in order to analyze the effects on human lung adenocarcinoma H460 cells. Changes in the rate of cell proliferation were detected among the Ctrl, rAd5-siCtrl and rAd5-siTrop2 groups following the siRNA interference. The results demonstrated that the proliferation rate and doubling time of H460 cancer cells was reduced following the silencing of Trop2, indicating that Trop2 promotes the proliferation of human lung adenocarcinoma H460 cells.

Trop2 has been demonstrated to promote the proliferation and differentiation of pancreatic tumor and intestinal tumor cells via regulation of the ERK signaling pathway (11). In addition, a previous study using mouse models of pancreatic and intestinal tumors revealed that high expression levels of Trop2 correlated positively with the expression of cyclin D1 and cyclin E, while a negative correlation was observed with P27 expression (20). Thus, Trop2 was shown to promote the proliferation and differentiation of pancreatic and intestinal tumor cells by regulating the ERK1/2 signaling pathway, and it was hypothesized that Trop2 may exert this effect in additional tumor types. The MAPK signal transduction pathway is closely associated with tumor cell proliferation. Raf/MEK/ERK is a key MAPK pathway, and among the most extensively studied pathways in the field of signal transduction. ERK is the key factor in the determination of cell fate following external stimulation, and is present as two forms; ERK1 and ERK2. ERK1/2 moves from the cytoplasm to the nucleus, where the protein phosphorylates a series of transcription factors, including c-Jun and c-Fos. The activation signal in the cytoplasm is subsequently transferred to the nucleus, forming a transcriptional complex that regulates gene expression, promotes cell proliferation and determines the end-stage differentiation or apoptosis of the cell. Cyclin D1 is highly expressed in numerous tumor types, and ERK is a crucial mediator of cyclin D1 expression (21). In addition, cyclin D1 binds to cyclin-dependent kinases, which subsequently promotes tumor cell proliferation (22).

The mechanisms underlying the effects of Trop2 on human lung adenocarcinoma H460 cell proliferation have not been previously reported. Therefore, the aim of the present study was to compare the protein expression levels of ERK-1 and cyclin D1 in Ctrl, rAd5-siCtrl and rAd5-siTrop2 groups of human lung adenocarcinoma H460 cells. Trop2 silencing was shown to downregulate the expression of ERK-1 and cyclin D1, indicating that the inhibition of human lung adenocarcinoma H460 cell proliferation and differentiation may be associated with the regulation of the ERK pathway. 
Invasion and metastasis are key processes in malignant tumor development, and are the primary factors for treatment failure and mortality in patients with cancer. The migration and invasion of tumor cells is a multi-step, continuous and active process. Tumor cells released from the primary site pass through extracellular matrix barriers and blood vessel walls to enter local and distant tissues (23). The application of siRNA in the targeting and silencing of key genes that affect the migration and invasion of tumor cells offers the opportunity for lung cancer gene therapy (23).

In the present study, Transwell chambers applied with Matrigel were used to observe the effect of inhibiting the expression of Trop2 on the migration and invasion of human lung adenocarcinoma $\mathrm{H} 460$ cells. No statistically significant difference was detected in the number of cells that invaded the Matrigel membrane when comparing the Ctrl group with the rAd5-siCtrl group. By contrast, significantly fewer cells in the rAd5-siTrop2 group were observed to traverse the Matrigel membrane compared with the Ctrl and rAd5-siCtrl groups. These results indicate that the suppression of Trop2 expression reduces the invasiveness of human lung adenocarcinoma $\mathrm{H} 460$ cells in vitro.

In conclusion, siRNA technology was employed to downregulate the expression of the Trop2 gene in human lung adenocarcinoma $\mathrm{H} 460$ cells. The downregulation of Trop2 was observed to inhibit the proliferation, migration and invasion of lung adenocarcinoma $\mathrm{H} 460$ cells in vitro. In addition, the mechanisms underlying the function of Trop2 in human lung adenocarcinoma $\mathrm{H} 460$ cells may involve the ERK pathway and the subsequent interactions with cyclin D1. These results provide novel experimental data for genetic studies investigating the function served by Trop2 in the progression of lung cancer.

\section{References}

1. Molina JR, Yang P, Cassivi SD, Schild SE and Adjei AA Non-small cell lung cancer: Epidemiology, risk factors, treatment, and survivorship. Mayo Clin Proc 83: 584-594, 2008.

2. Jemal A, Bray F, Center MM, Ferlay J, Ward E and Forman D: Global cancer statistics. CA Cancer J Clin 61: 69-90, 2011.

3. Yang L, Parkin DM, Ferlay J, Li L and Chen Y: Estimates of cancer incidence in China for 2000 and projections for 2005 Cancer Epidemiol Biomarkers Prev 14: 243-250, 2005.

4. Yamamoto C,Basaki Y,Kawahara A, et al: Loss of PTEN expression by blocking nuclear translocation of EGR1 in gefitinib-resistant lung cancer cells harboring epidermal growth factor receptor-activating mutations. Cancer Res 70: 8715-8725, 2010.

5. Jiang A, Gao X, Zhang D, Zhang L and Lu H: Expression and clinical significance of the Trop- 2 gene in advanced non-small cell lung carcinoma. Oncol Lett 6: 375-380, 2013.

6. Gelsomino F, Rossi G and Tiseo M: Clinical implications and future perspectives in testing non-small cell lung cancer (NSCLC) for anaplastic lymphoma kinase (ALK) gene rearrangements. J Thorac Dis 7: 220-223, 2015.
7. Thomas A, Lee JH, Abdullaev Z, et al: Characterization of fibroblast growth factor receptor 1 in small-cell lung cancer. J Thorac Oncol 9: 567-571, 2014.

8. Calabrese G, Crescenzi C, Morizio E, Palka G, Guerra E and Alberti S: Assignment of TACSTD1 (alias TROP1, M4S1) to human chromosome $2 \mathrm{p} 21$ and refinement of mapping of TACSTD2 (alias TROP2, M1S1) to human chromosome 1p32 by in situ hybridization. Cytogenet Cell Genet 92: 164-165, 2001.

9. Taylor JT, Zeng XB, Pottle JE, et al: Calcium signaling and T-type calcium channels in cancer cell cycling. World J Gastroenterol 14: 4984-4991, 2008.

10. Nelsen CJ, Kuriyama R, Hirsch B, et al: Short term cyclin D1 overexpression induces centrosome amplification, mitotic spindle abnormalities, and aneuploidy. J Biol Chem 280: 768-776, 2005.

11. Cubas R, Zhang S, Li M, Chen C and Yao Q: Trop2 expression contributes to tumor pathogenesis by activating the ERK MAPK pathway. Mol Cancer 9: 253, 2010.

12. Jiang HF and Bo JJ: Trophoblastic cell surface protein 2 and tumor. Guo Ji Zhong Liu Xue Za Zhi 39: 96-98, 2012 (In Chinese).

13. Ohmachi T, Tanaka F, Mimori K, Inoue H, Yanaga $\mathrm{K}$ and Mori M: Clinical significance of TROP2 expression in colorectal cancer. Clin Cancer Res 12: 3057-3063, 2006.

14. Fong D, Spizzo G, Gostner JM, et al: TROP2: A novel prognostic marker in squamous cell carcinoma of the oral cavity. Mod Pathol 21: 186-191, 2008.

15. Fong D, Moser P, Krammel C, et al: High expression of TROP2 correlates with poor prognosis in pancreatic cancer. Br J Cancer 99: 1290-1295, 2008

16. Kobayashi H, Minami Y, Anami Y, Kondou Y, Iijima T, Kano J, Morishita Y, Tsuta K, Hayashi S and Noguchi M; IASLC International Staging Committee: Expression of the GA733 gene family and its relationship to prognosis in pulmonary adenocarcinoma. Virchows Arch 457: 69-76, 2010.

17. Pak MG, Shin DH, Lee $\mathrm{CH}$ and Lee MK: Significance of EpCAM and TROP2 expression in non-small cell lung cancer. World J Surg Oncol 10: 53, 2012.

18. Gu YP, Zhao YG, Zhu YB, Zhang GB and Huang JA: Primary study of expression of TROP-2 in human pulmonary adenocarcinoma and cell lines. Zhong Liu Fang Zhi Za Zhi 20: 342-346, 2013 (In Chinese)

19. Jiang AG, Lu HY, Zhang DG, Zhang LX and Gao XY: Short hairpin RNA targeting AKT1 and PI3K/p85 suppresses the proliferation and self renewal of lung cancer stem cells. Mol Med Rep 12: 363-370, 2015.

20. Shen YH, Godlewski J, Bronisz A, et al: Significance of 14-3-3 self-dimerization for phosphorylation-dependent target binding. Mol Biol Cell 14: 4721-4733, 2003

21. Tong XD, Liu HX, Zhao HR, et al: The role of Smad4 and MAPK proteins in signal transduction pathway in non-small cell lung cancer. Zhonghua Zhong Liu Za Zhi 28: 741-745, 2006 (In Chinese).

22. Lebeau A, Unholzer A, Amann G, et al: EGFR, HER-2/neu, cyclin D1, p21 and p53 in correlation to cell proliferation and steroid hormone receptor status in ductal carcinoma in situ of the breast. Breast Cancer Res Treat 79: 187-198, 2003.

23. Martin MD and Matrisian LM: The other side of MMPs: Protective roles in tumor progression. Cancer Metastasis Rev 26: 717-724, 2007. 